\title{
COMPETIÇÃO INICIAL ENTRE Cyperus esculentus E ARROZ IRRIGADO EM CONDIÇÕES DE CASA-DE-VEGETAÇÃO ${ }^{1}$
}

\author{
ERASMO E. A. L. ${ }^{2}$, PINTO L. P. ${ }^{3}$, TERRA, M. ${ }^{3}$, DIDONET, J. ${ }^{2}$ e PELUZIO, J. M. ${ }^{2}$
}

\section{RESUMO}

Com o objetivo de avaliar o efeito competitivo de Cyperus esculentus sobre o crescimento inicial da cultura do arroz irrigado, foi instalado um experimento em casa-devegetação, na Estação Experimental da Faculdade de Agronomia - UNITINS, no munícipio de Gurupi-TO. O delineamento estatístico utilizado foi um fatorial $5 \times 4$ com três repetições completamente casualizado. Os tratamentos constaram de cinco períodos de convivência do arroz com C. esculentus (15, 25, 35, 45 e 60 dias após a emergência da cultura) e quatro densidades de $C$. esculentus $(0,2,4$ e 8 tubérculos/vaso), correspondentes a $0 ; 71 ; 142$ e 286 plantas de $C$. esculentus por $\mathrm{m}^{2}$, respectivamente. No final de cada período foram avaliados na cultura do arroz os seguintes parâmetros: matéria seca de plantas/vaso; área foliar/planta; matéria seca de perfilhos/vaso; n. ${ }^{\circ}$ de perfilhos/vaso e altura média de plantas. Os resultados mostraram que o efeito da presença de C. esculentus foi mais marcante nas densidades de quatro e oito tubérculos/vaso a partir dos 35 dias de convivência. O parâmetro mais afetado foi a matéria seca de plantas/vaso, como resultado do decréscimo do número de perfilhos/vaso. A altura das plantas de arroz irrigado não foi afetada pela presença da planta daninha em nenhum período de convivência.

Palavras chave: Planta daninha, Oryza sativa, interferência.

\section{ABSTRACT \\ Competition between Cyperus esculentus and irrigated rice under green house conditions}

With the objective of evaluating the competitive effect of Cyperus esculentus on the growth and production of the culture of irrigated rice, experiments were installed in green house, in the Experimental Station of the School of Agronomy - UNITINS, in Gurupi, State of Tocantins, Brazil. The statistical design used in the experiments was a factorial plot $5 \times 4$ with three replications, completely randomized. The treatments consisted of five periods of coexistence of the rice with Cyperus esculentus $(15,25,35,45$ and 60 days after the emergency D.A.E, of the rice) and four densities of $C$. esculentus ( $0,2,4$ and 8 tuber/pots) corresponding to $0 ; 71 ; 142$ e 286 plants of Cyperus $/ \mathrm{m}^{2}$, respectively. In the end of each period the following parameters in the rice culture were appraised: dry matter of plants/pot; leaf area/plant; tiller/pot dry matter; tiller/pot number and height of plants. The results showed that the effect of the of $C$. esculentus, was more pronounced in the densities of four and eight tuber/pot, in the periods of coexistence of 45 and 60 D.A.E. The most affected parameter was the dry matter of plant/pot, as a result of the reduction of the tiller/pot number. The height of the plants of irrigated rice was not affected by the presence of the weed in any coexistence period.

Key words : Weed, Oryza sativa, interference.

\footnotetext{
${ }^{1}$ Recebido para publicação em 07/07/99 e na forma revisada em 15/02/2000.

${ }^{2}$ Prof. da Fundação Universidade do Tocantins. Av. Madrid, Q. 6, Lt. 8 e 9, Jardim Sevilha, CEP: 77410-470.

${ }^{3}$ Graduando em Agronomia na Fundação Universidade do Tocantins e bolsista do PIBIC/CNPQ.
} 


\section{INTRODUÇÃO}

As condições ecológicas próprias a ecossistemas tipo várzea contribuem para uma grande proliferação de diversas espécies de plantas, que surgem espontaneamente junto às lavouras, a exemplo da cultura do arroz irrigado. Estas plantas, denominadas de daninhas, competem pelos fatores de crescimento, a saber: luz, água, nutrientes e espaço, sendo que a sua presença e falta de controle no tempo adequado, tem ocasionado grandes perdas no produto colhido e elevados custos na produção.

$O$ grau de competição entre as comunidades infestantes e as culturas agrícolas vai depender de fatores ligados à própria comunidade infestante (composição específica, densidade e distribuição), à cultura (espécie ou variedade, espaçamento e densidade de plantio) e à época de extensão do período de convivência, podendo estes fatores serem alterados pelas condições edáficas, climáticas, e tratos culturais (Pitelli, 1985).

A composição florística dessas comunidades infestantes variam conforme a região geográfica, entretanto, dentro das várias famílias e espécies de plantas daninhas, que invadem a cultura do arroz irrigado a nível mundial, são comuns aquelas pertencentes as famílias Poaceae e Cyperaceae, com mais de 60 e 50 espécies, respectivamente (Smith e Morody, citados por SMITH, 1983). No Brasil, esta afirmativa não é diferente, sendo que, especificamente na região do Formoso da Araguaia, Estado do Tocantins, destacam-se as espécies da familia das Cyperaceas, em especial Cyperus esculentus e Cyperus iria.

Um dos fatores mais determinantes no grau de competição é a densidade das espécies presentes, desta maneira, o incremento na densidade das plantas daninhas aumenta as perdas na cultura do arroz irrigado, variando segundo a espécie. Amaral (1995) verificou que 40 plantas de Cyperus esculentus $/ \mathrm{m}^{2}$ convivendo com a cultura de arroz irrigado até a colheita, ocasionou uma redução na produção de grãos de $38 \%$. Já, Smith (1983) trabalhando com a espécie daninha Echinochloa crusgalli, constatou que densidades de 11,54 e 269 plantas $/ \mathrm{m}^{2}$ reduziam a produção de arroz em 25, 49 e $89 \%$, respectivamente.

Associado a densidade, a época e duração do período em que a cultura e a comunidade infestante coabitam, modificam a intensidade de competição. Ishy e Lovato (1974) citam que o arroz irrigado, nas condições específicas onde foi conduzido o trabalho, apresentou um período crítico de competição de 15 a 45 dias, após a emergência. Deuber e Forster (1972) recomendam que a cultura do arroz irrigado deve ficar livre das plantas daninhas em torno de 32 dias, de maneira a não sofrer queda na produção.

Portanto, o conhecimento adquirido por meio de estudos de competição, realizados tanto no campo como em casa-de-vegetação, com espécies frequentes em lavouras de arroz irrigado, a exemplo do realizado no presente projeto, servem de apoio tanto como alerta dos erros cometidos com controles tardios, bem como auxílio nas tomadas de decisões no manejo de plantas daninhas. Assim, o presente trabalho objetivou avaliar o efeito da densidade e períodos de convivência de Cyperus esculentus, no crescimento inicial da cultura do arroz irrigado.

\section{MATERIAL E MÉTODOS}

O presente projeto foi conduzido sob condições de casa-de-vegetação na Estação Experimental da Universidade do Tocantins (Centro Universitário de Gurupi), localizado no Município de Gurupi/TO, situado geograficamente entre os paralelos $11^{\circ}$ e $12^{\circ}$ de Latitude Sul e os meridianos $49^{\circ}$ e $50^{\circ}$ de Longitude Oeste.

O experimento constituiu-se de uma fatorial 4 x 5 com três repetições, disposto em um delineamento inteiramente casualizado, onde os tratamentos constaram de quatro densidades de Cyperus esculentus ( $0,2,4,8$ planta/vaso) correspondentes a $0 ; 71 ; 142$ e 286 plantas de 
Cyperus por $\mathrm{m}^{2}$, respectivamente e cinco períodos de convivência $(15,25,35,45$ e 60 dias após a emergência).

As parcelas foram constituídas por vasos de cerâmica, com capacidade de cinco litros e 24,5 $\mathrm{cm}$ de diâmetro. O substrato de crescimento utilizado, foi solo coletado a $10 \mathrm{~cm}$ de profundidade de uma área de várzea característica, seco ao ar e peneirado em tamis de dois milímetros.

A umidade do solo foi mantida através de irrigação freqüente, até os 25 dias iniciais após a emergência, sendo posteriormente mantido em saturação constante.

A semeadura da cultura do arroz cultivar Metica 1, foi realizada no dia 02/12/1996, depositando-se um número maior de sementes, procedendo-se a um desbaste cinco dias após a emergência, de forma a se obter 11 plantas de arroz por vaso. No mesmo dia foram constituídos os tratamentos relativos a densidade de C. esculentus, plantando-se o número de tubérculos (tamanho padronizado) correspondente por vasos.

Ao final de cada período de convivência entre C. esculentus e a cultura do arroz, os vasos foram desmontados, sendo avaliados na cultura os seguintes parâmetros: matéria seca e número de perfilhos/vaso; matéria seca da parte aérea/vaso; altura das plantas e área foliar/planta. Após o corte das plantas rente ao solo, destacaram-se a seguir as lâminas foliares medindo-se o seu comprimento e largura máxima, para cálculo da área foliar $(\mathrm{AF}) \mathrm{em} \mathrm{cm}^{2}$, através da fórmula $\mathrm{AF}=0,82 \times(\mathrm{CxL})$ segundo Bhan et. al. (1966). Os colmos, bainhas, e as lâminas foliares foram lavadas e secas em estufa com circulação forçada de ar a $70^{\circ} \mathrm{C}$ por um período de três dias, sendo pesados posteriormente em balança de precisão.

Os resultados foram submetidos a análise de variância pelo teste $\mathrm{F}$ e teste de comparação de médias (Tukey 5\%). Na presença de interações significativas foram realizados os respectivos desdobramentos.

\section{RESULTADOS E DISCUSSÃO}

Na Figura 1 estão expressas as curvas de regressão correspondentes ao acúmulo de matéria seca das plantas de arroz irrigado em função do período de convivência com diferentes densidades de Cyperus esculentus.

Observou-se que em todas as densidades, com exceção do tratamento de oito tubérculos/vaso, o acúmulo de matéria seca das plantas de arroz ajustou-se a curvas tipo quadrática, enquanto que no tratamento de menor densidade de tubérculos o modelo foi linear.

As plantas de arroz evidenciaram um incremento no acúmulo de matéria seca praticamente similar até os 35 dias após a emergência (D.A.E), a partir do qual ficou mais evidente o efeito competidor de Cyperus esculentus, demonstrado pelo decréscimo crescente na matéria seca por vaso, com o aumento da densidade da planta daninha. Estes decréscimos foram na ordem de 17 e $51 \%$, quando comparam-se, a matéria seca de plantas de arroz crescidas em vasos com densidades de oito tubérculos e aquelas sem a presença da Cyperacea (0 tubérculos/vaso), em períodos de 45 e 60 D.A.E., respetivamente.

As densidades de dois e quatro tubérculos de Cyperus esculentus por vaso não evidenciaram praticamente nenhuma diferença em relação a matéria seca de plantas de arroz nos diferentes períodos de convivência, com exceção aos 60 D.A.E., onde constatou-se uma redução em média de $13 \%$, quando comparada ao tratamento de zero tubérculos/vaso. Ao analisar-se a evolução da área foliar das plantas de arroz irrigado em função do período de convivência com diferentes densidades de Cyperus esculentus (Figura 2), verifica-se um incremento natural ao longo do tempo, consequente ao crescimento das plantas; no entanto, apresentaram diferenças na magnitude das mesmas a partir dos 35 D.A.E.. A partir deste período, constatam-se decréscimos conforme aumento na densidade de tubérculos de Cyperus esculentus por vaso, sendo que as maiores reduções $(27 \%)$ foram verificadas aos 60 D.A.E. de convivência, ao comparar-se os tratamentos de oito e zero tubérculos/vaso. 

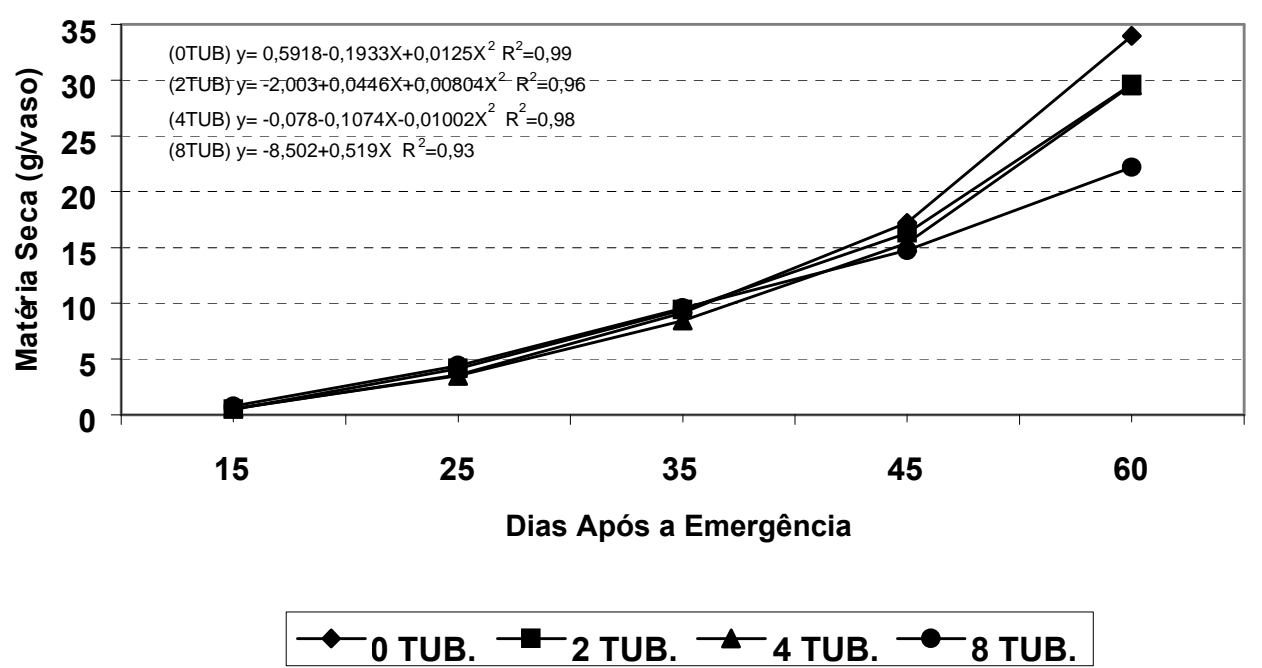

FIGURA 1. Curvas de regressão da evolução do acúmulo de matéria seca (parte aérea) de plantas de arroz irrigado em função do período inicial de convivência e densidades de C. esculentus.

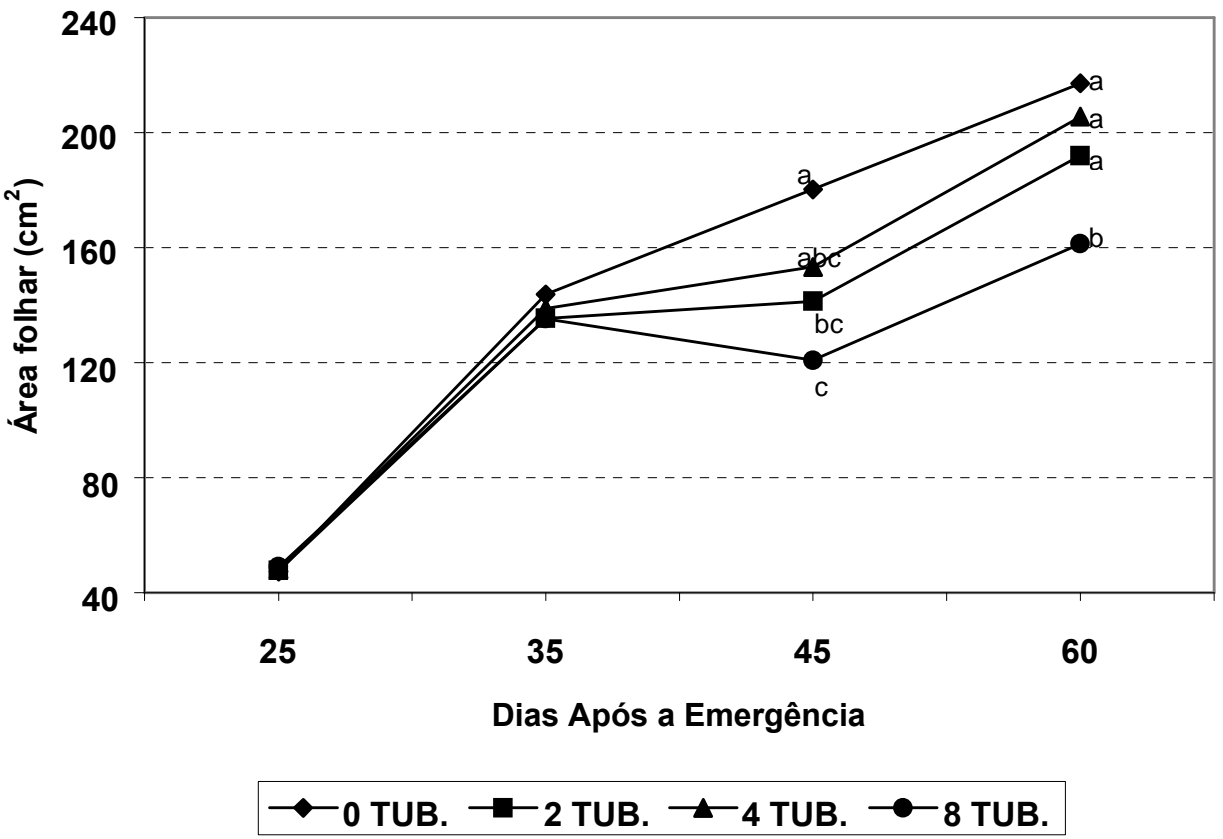

FIGURA 2. Variação da área foliar de plantas de arroz irrigado em função do período inicial de convivência com diferentes densidades de C. esculentus. 
O comportamento do número de perfilhos de arroz/vaso, ao longo do período experimental, de plantas desenvolvidas junto a densidades variáveis de Cyperus esculentus por diferentes períodos, pode ser analisado na Figura 4.

Verifica-se que o número de perfilhos por vaso apresentou-se praticamente estável em todos os tratamentos onde a Cyperaceae se fazia presente, enquanto no tratamento onde as plantas de arroz se desenvolveram sozinhas (zero tubérculos/vaso) ocorreu um incremento ao longo do período. A presença de quatro e oito tubérculos/vaso reduziram o número de perfilhos em 15 e 26\%, respectivamente, quando conviveram com a cultura 45 dias. A convivência por um período de 60 dias elevou essa redução em $34 \%$ em média.

Na Figura 4 está representado o peso seco de perfilhos de arroz/vaso, de plantas desenvolvidas em associação com diferentes densidades de Cyperus esculentus em diferentes períodos.

Analisando-se a figura, observa-se que o maior incremento no peso seco de perfilhos aconteceu a partir dos 45 D.A.E., sendo que o tratamento com densidade de oito tubérculos de Cyperus esculentus/vaso, diferiu estatisticamente dos demais tratamentos. Neste, foi verificado uma redução no peso seco de perfilhos na ordem de 37 e 39\%, após 45 e 60 dias de convivência, respectivamente, quando comparado ao tratamento sem a presença da Cyperacea (zero tubérculos/vaso) . Nos outros dois tratamentos (dois e quatro tubérculos/vaso), o decréscimo foi somente observado aos 60 dias de convivência (17\% em média).

A altura das plantas de arroz não foi afetada pela presença da Cyperacea em nenhum período (Figura 5).

Realizando-se uma análise conjunta dos diferentes parâmetros avaliados na cultura do arroz irrigado, observa-se claramente a definição de algumas respostas em comum. O efeito competitivo da Cyperaceae foi praticamente evidenciado a partir dos 35 dias após a emergência da cultura com decréscimos crescentes até o final do período experimental, sendo mais evidente nas maiores densidades (quatro e oito tubérculos/vaso) e nos períodos mais prolongados de convivência. Este decréscimo foi mais expressivo no acúmulo de matéria seca de plantas por vaso, como conseqüência direta da redução do número de perfilhos. Esta resposta, foi também constatado por Dáros (1988) o qual verificou uma redução de $29 \%$ no número de perfilhos por $\mathrm{m}^{2}$ em plantas de arroz irrigado, que se desenvolveram junto com uma comunidade diversa de plantas daninhas.

$\mathrm{O}$ maior efeito supressor exercido pela planta daninha no período citado anteriormente, pode ser explicado pelo fato de que nesse período conjugaram-se duas épocas críticas do crescimento de ambas espécies conviventes. As plantas de arroz encontravam-se na época de maior intensidade de crescimento vegetal representado pelo surgimento de perfilhos, normalmente compreendido entre 20 a 60 dias após a emergência da cultura, enquanto que, a planta daninha apresentava-se no início do período de florescimento. A vantagem da Cyperacea pode estar relacionada além da sua agressividade natural, como a alta capacidade na competição por nutrientes, também ao possível efeito alelopático caraterístico desta espécie ( Drost \& Doll, 1980). Neste período coincidiu ainda a aplicação de adubos nitrogenados, o que provavelmente beneficiou em maior grau à Cyperaceae.

Os resultados alertam às consequências no atraso do controle desta espécie, incluindo a entrada tardia da água na cultura. Foi observado que um atraso de 10 dias no período compreendido entre 35 e 45 dias de convivência, ocasionou um aumento na redução no número de perfilhos de 13 a $27 \%$, numa densidade da Cyperaceae de oito tubérculos/vaso, que corresponde a 286 plantas $/ \mathrm{m}^{2}$. Certamente a redução do número de perfilhos acarretará em decréscimo na produção de grãos, conforme constatado por Amaral (1995), o qual verificou que 40 plantas $/ \mathrm{m}^{2}$ de C. esculentus convivendo com a cultura de arroz irrigado por todo o ciclo provocou reduções na ordem de $38,5 \%$. 


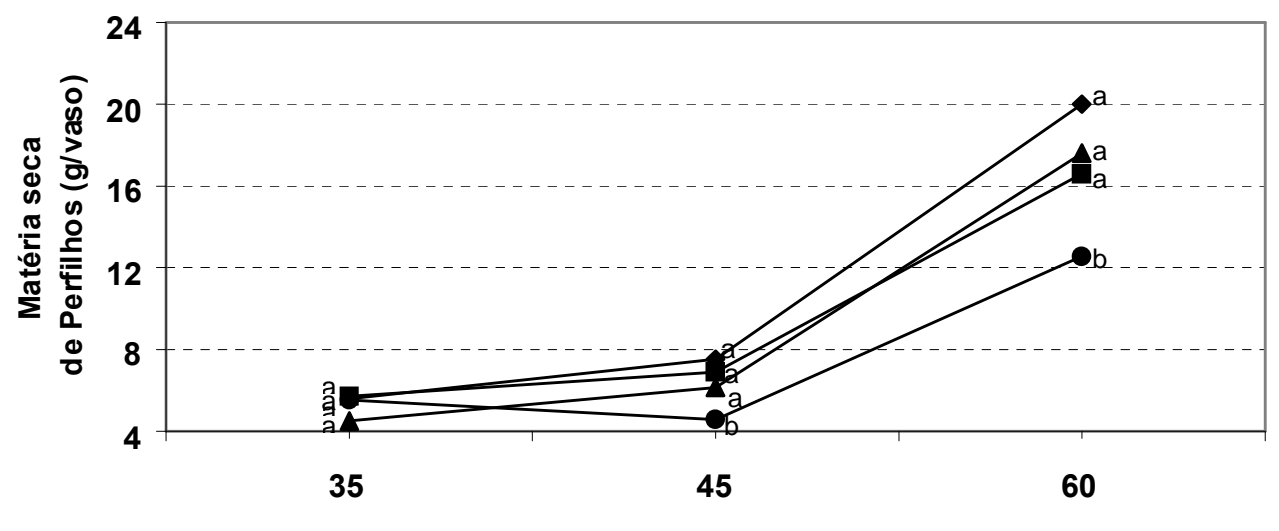

Dias Após a Emergência

0 TUB.

2 TUB.

4 TUB.

8 TUB.

FIGURA 3. Matéria seca de perfilhos de arroz irrigado em função do período de convivência inicial com diferentes densidades de C. esculentus.

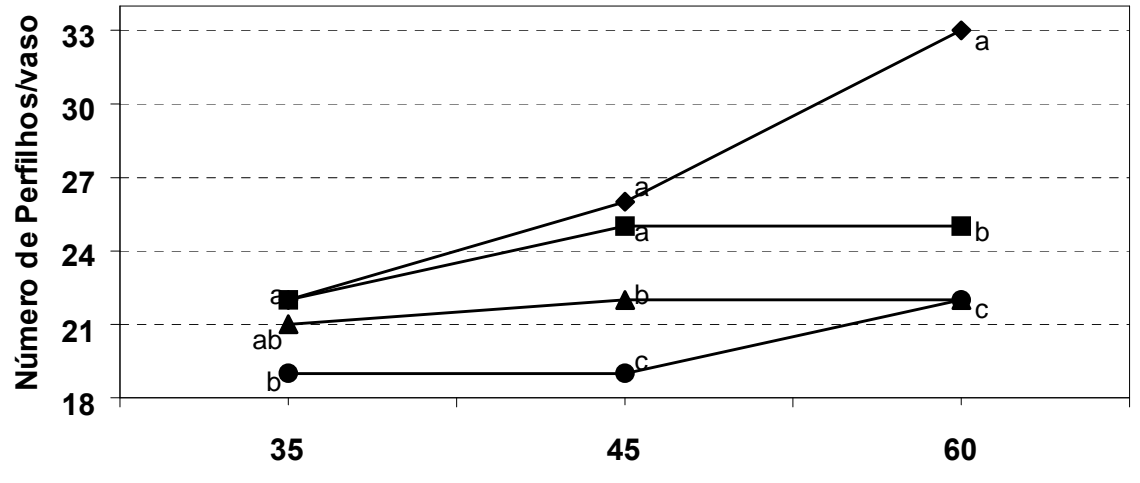

Dias Após a Emergência

0 TUB. $\rightarrow 2$ TUB. $\leftarrow 4$ TUB

8 TUB.

FIGURA 4. Número de perfilhos de arroz irrigado em função do período inicial de convivência com diferentes densidades de C. esculentus. 


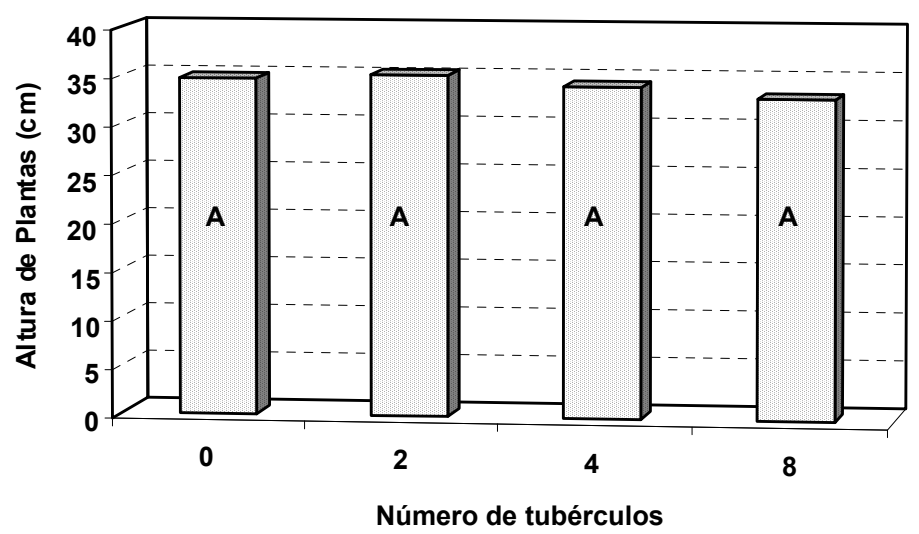

FIGURA 5. Altura de plantas de arroz irrigado em função da densidade de $C$. esculentus.

\section{LITERATURA CITADA}

AMARAL, A.S. Controle químico de ciperáceas na cultura do arroz irrigado. Lavoura Arrozeira, Porto Alegre, v. 48, n. 420, p.36, 1995.

BHAN, V.M. et al. Measerument of leaf area of rice. Agron. J., v 58, p.454, 1966.

\section{DÁROS, R. Invasoras na cultura de arroz} irrigado. Campo Grande-MS.: EMPAER, 1988. 50 p. (Boletim de Pesquisa, 5).

DEUBER, R; FORSTER, R. Cultivo e competição ervas na cultura do arroz (Oryza sativa). In: SEMINÁRIO BRASILEIRO DE HERBICIDAS E ERVAS DANINHAS, 9, Campinas, 1972. Resumos. Campinas, SBHED, p. 20, 1972.
DROST, D.C. \& DOLL, J.D. The allelophatic effect of yellow nutsedge (Cyperus esculentus) on corn (Zea mays) and soyabean (Glycine max). Weed Sci., v. 28, p.229-33, 1980.

ISHIY, T; LOVATO, L.A. Influências das ervas daninhas na produção de arroz. Lavoura Arrozeira. Porto Alegre, v.27, n. 278, p.4850, 1974.

PITELLI R. A. Interferências de plantas daninhas em culturas agrícolas. Inf. Agropec.. Belo Horizonte, V. 11, no 129, p. 16-27, 1985.

SMITH, R. J. Weeds of major economic importance in rice and yield losses due to weed competition. In: CONFERENCE ON WEED CONTROL IN RICE, Philipines, Los Baños, IRRI. p. 19-36, 1983. 